\title{
Ueber Hemimellithsaure;
}

von C. Graebe und M. Leonhardt.

Die Hemimellithsäure (1,2,3-Benzoltricarbonsăure) wurde von A, Baeyer ${ }^{1}$ ) gelegentlich seiner Untersuchung der Mellithsäure (1870) entdeckt aber nicht eingehend studirt, da sie sich bei der Einwirkung von Schwefelsäure auf Hydroisopyromellithsäure nur in geringer Menge bildet. Auch muss die von Baeyer beschriebene Säure, wie aus den Schmelzpunktsangaben hervorgeht, noch Phtalsäure enthalten haben. Später (1893) theilte H. Hosaeus mit, dass sie auch aus $\beta$-Oxynaphtoësäure durch Oxydation entstehe, doch ist diese Angabe nach J. Schmid und nach M. Schöpf unrichtig ${ }^{2}$ ). In demselben Jabre fanden Graebe nnd Bossel ${ }^{8}$ ), dass man die Hemimellithsäure vollkommen rein aus Acenaphten erhalten kann. Diese Beobachtung machte es nun möglich, sie in grösserer Menge darzustellen und genauer zu untersuchen. Inzwischen hat $B$. Jeitelest) angegeben, dass sie als $0 x y-$ dationsproduct der Isochinolinearbonsäure auftrete, doch lässt sich aus den mitgetheilten Beobachtungen nicht beurtheilen, ob wirklich Hemimellithsäure vorlag.

Wir haben nun in erster Linie die Darstellungsmethode ausgearbeitet, dann die Esterbildung, die Condensationsproducte mit Benzol nach Friedel-Craft'scher Reaction sowie die entsprechenden Fluoresceïne näher untersucht.

\section{Darstellung der Hemimellithsäure.}

Wie in der vorhergehenden Arbeit mitgetheilt ist, wird die freie Phenylglyoxyldicarbonsäure durch Permanganat leicht und ganz quantitativ zu Hemimellithsäure oxydirt. Zur Ge-

\footnotetext{
1) Diese Annalen Suppl. 7, 31.

z) Ber. d. deutseh. chem. Ges. 26, 666, 1114 und 1123.

3) Ber. d. deutsch. chem. Ges. 26, 1797.

†) Monatshefte f. Chem. 15, 816 .
} 
winnung der letzteren ist es nun unnöthig, die Phenylglyoxyldicarbonsäure zu isoliren und kann man die Darstellung dadurch sehr vereinfachen, dass man zuerst die Naphtalsäure in alkalischer Lösung oxydirt, dann das Alkali durch Schwefelsüure in neutrale Sulfate verwandelt und von neuem mit Kaliumpermanganat behandelt. $\mathrm{Zu}$ diesem Zweck ist es nöthig, das zum Auflösen der Naphtalsäure benutzte Natron sowie das Permanganat genau abzuwägen, um die berechnete Menge Schwefelsäure zufügen zu können. Hat man die Naphtalsäure frisch aus reinem Acenaphten dargestellt, so kann man bei der Berechnung der Permanganatmenge, welche erforderlich ist, von der Annahme ausgehen, dass zehn Theile Acenaphten 11,5 Theile Naphtalsäureanhydrid liefern und man kann direct das aus dem Kohlenwasserstoff durch Erhitzen mit Natriumchromat und Eisessig erhaltene Product verwenden. Auch lässt sich bei frisch ausgefällter Naphtalsäure, welche gewöhnlich aus einem Gemenge von Saure und Anhydrid besteht, durch Erhitzen einer Probe auf $150-160^{\circ}$ rasch bestimmen, wieviel Anhydrid sie entspricht.

Zehn Theile Naphtalsäureanhydrid werden mit vier Theilen Aetznatron, welche in 40-50 Theilen Wasser gelöst sind, auf dem Wasserbade erhitzt und 48 Theile Kaliumpermanganat in concentrirter siedender wässriger lösung zugegeben und zwar so rasch als Entfärbung eintritt. Wird die Oxydation träger, so fugt man den Rest des Oxydationsmittels hinzu und erwärmt noch zwei bis drei Stunden auf dem Wasserbade. Darauf wird durch Alkohol das überschüssige Permanganat reducirt, heiss filtrirt und mit heissem Wasser das Mangandioxyd ausgewaschen. Man giebt die dem Natron und dem gebildeten Kali genau entsprechende Menge Schwefelsäure hinzu, die man ziemlich concentrirt anwenden kann und die für obige Mengen 19,8 Theile $\mathrm{H}_{2} \mathrm{SO}_{4}$ enthalten muss. Man oxydirt dann mit einer Lösung von fünf Theilen Kaliumpermanganat. Die Farbe verschwindet sofort; aber erst nachdem der grössere Theil des Permanganats zugegeben ist, beginnt 
die Ausscheidung von Mangandioxyd. Sowie alles Oxydationsmittel entfärbt ist, wird filtrirt und soweit eingedampft, dass das Volumen der Flüssigkeit ungefähr $250 \mathrm{ccm}$ für $10 \mathrm{~g}$ angewandten Naphtalsäureanhydrids beträgt. Beim Erkalten der Lösung beginnt sofort die Krystallisation des Monokaliumsalzes der Hemimellithsäure, welche sich fast vollständig und ganz rein ausscheidet. Durch Eindampfen des Filtrats, Versetzen mit Salzsäure und Ausziehen mit Aether lässt sich noch etwas aber nur wenig Hemimellithsäure erhalten. Aus $10 \mathrm{~g}$ Naphtalsäureanhydrid wurden $9,8-10 \mathrm{~g}$ bei $100^{\circ}$ getrocknetes Kalisalz, welche $8,6-8,8 \mathrm{~g}$ Hemimellithsäure (wasserfrei) entsprechen und aus der Mutterlauge noch $0,5-0,7 \mathrm{~g}$ freie Säure erhalten. Theoretisch können aus $10 \mathrm{~g}$ Naphtalsäureanhydrid $11,1 \mathrm{~g}$ Hemimellithsäure entstehen. Da man aus 100 Theilen Acenaphten 115-116 Theile Naphtalsäureanhydrid erhält, so liefern dieselben also ungefähr 105 Theile Hemimellithsäure.

Um die Hemimellithsäure aus dem Kaliumsalz zu gewinnen hatten wir anfangs dieses in einer möglichst geringen Menge heissem Wasser gelöst und dann einen sehr grossen Ueberschuss ganz concentrirter Salzsäure zugegeben. Die dann beim Erkalten auskrystallisirende Säure entbält aber fast immer noch Kalisalz beigemengt und man muss, um ganz aschenfreie Säure zu erhalten, diese Operation wenigstens noch einmal wiederholen. Man kann auch das Kaliumsalz durch Erwärmen in einer 25-30 procentigen Salzsäure auflösen; aber auch hierbei scheidet sich meist die Hemimellithsäure noch kaliumhaltig aus. Wendet man zum Zersetzen des Salzes eine $30-40$ procentige Schwefelsäure an, so wird die Säure sofort reiner. In beiden Fällen erhält man aber grosse Mengen stark saurer Mutterlaugen, aus denen die in Lösung gebliebene Säure nur mühsam gewonnen werden kann. Zweckmässiger ist es daher, das Monokaliumsalz zuerst in das neutrale Baryumsalz zu verwandeln und dieses mit der berechneten Menge oder einem kleinen Ueberschuss von Schwefelsäure zu zersetzen. Man löst das Kaliumsalz unter Zusatz von Ammoniak in Alnalen der Chemie 290. Bd. 
möglichst wenig heissem Wasser, giesst diese Lösung in eine warme Lösung von Chlorbaryum und erwärmt noch einige Zeit. Das Baryumsalz scheidet sich krystallisirt ans. Man kann sofort oder nach dem Erkalten filtriren, da dasselbe in heissem Wasser kaum mehr löslich ist wie in kaltem. Es bleibt nor sehr wenig Salz in Lösung. Nach den unten angegebenen Löslichkeitsbestimmungen enthalten $100 \mathrm{ccm}$ Wasser von $25^{\circ}$ soviel Baryumsalz, als $0,08 \mathrm{~g}$ Hemimellithsäure entspricht. Das Baryumsalz wird gut aber mit möglichst wenig Wasser ausgewaschen und daun noch feucht mit verdünnter Schwefelsäure zersetzt, deren Menge man nach dem angewandten Kaliumsalz berechnet. Für $10 \mathrm{~g}$ hemimellithsaures Monokalium (bei $100-110^{\circ}$ getrocknet) benutzt man zum Fällen ungefähr $20 \mathrm{~g}$ krystallisirtes und in $100 \mathrm{ccm}$ Wasser gelöstes Chlorbaryum und zum Zersetzen des Niederschlags eine verdünnte Schwefelsäure, welche $5,9 \quad \mathrm{~g} \mathrm{H}_{2} \mathrm{SO}_{4}$ enthält. Man concentrirt das Filtrat, bis die Hemimellithsäure zu krystallisiren beginnt. Die Mutterlauge von der ausgeschiedenen Säure dampft man auf dem Wasserbade ein, und wenn etwas überschüssige Schwefelsäure vorhanden war, entfernt man diese durch Pressen oder Absaugen auf einem Thonteller.

\section{Figenschaften der Hemimellithsäure.}

Die Hemimellithsäure krystallisirt aus wässriger Lösung in Tafeln, welche sehr schön ausgebildet sind und die man von beträchtlicher Grösse erhalten kann. In heissem Wasser ist sie sehr reichlich löslich, viel weniger in kaltem. Eine bei $19^{\circ}$ gesättigte Lösung enthält auf $100 \mathrm{~g}$ Wasser $3,15 \mathrm{~g}$ Hemimellithsäure; Salzsäure vermindert die Löslichkeit in Wasser. Aether löst sie ziemlich reichlich.

Sowohl aus Wasser, Aether oder starker Salzsäure krystallisirt sie mit zwei Molekülen Wasser, welches schon im Exciccator über Schwefelsäure und rasch bei $100^{\circ}$ fortgeht. Folgende Analysen beziehen sich auf die obigen drei Lösungsmittel. 
Graebe und Leonhardt, Ueber Hemimellithsäure. 221

$1,1356 \mathrm{~g}$ verloren $0,1656 \mathrm{H}_{2} \mathrm{O}$.

$0,1272 \mathrm{~g} \quad, \quad 0,0186 \mathrm{H}_{2} \mathrm{O}$.

$0,3470 \mathrm{~g} \quad, \quad 0,0502 \mathrm{H}_{2} \mathrm{O}$.

$\begin{array}{ccccc} & \text { Perechnet für } & & \text { Gefunden } \\ & \mathrm{C}_{8} \mathrm{H}_{8} \mathrm{O}_{6}+2 \mathrm{H}_{2} \mathrm{O} & & & \\ \mathrm{H}_{2} \mathrm{O} & \mathbf{1 4 , 6 4} & 14,58 & \mathbf{1 4 , 6 0} & \mathbf{1 4 , 4 8}\end{array}$

Dass die nach obiger Darstellung erhaltene Hemimellithsäure rein ist, geht aus den Verbrennungen und den Schmelzpunktsbestimmungen hervor.

$0,1764 \mathrm{~g}$ gaben $0,3320 \mathrm{CO}_{2}$ und $0,0443 \mathrm{H}_{2} \mathrm{O}$.

$0,1130 \mathrm{~g} \quad, \quad 0,2120 \mathrm{CO}_{2} \quad, \quad 0,0298 \mathrm{H}_{3} \mathrm{O}$.

\begin{tabular}{cccc} 
& Berechnet & \multicolumn{2}{c}{ Gefunden } \\
$\mathrm{C}$ & 51,43 & 51,33 & 51,25 \\
$\mathrm{H}$ & 2,80 & 2,80 & 2,93
\end{tabular}

Beim Erbitzen tritt bei $190^{\circ}$ Schmelzen unter Anbydridbildung ein, und nachdem man so lange anf $190-200^{\circ}$ erhitzt hat, bis die Wasserabspaltung aufgehört hat, ist der Schmelzpunkt gestiegen und liegt dann bei $196^{\circ}$. Dass bei $200^{\circ}$ sich die Hemimellithsäure glatt. in ihr Anhydrid verwandelt, beweisen folgende Analysen.

$1,3780 \mathrm{~g}$ bej $110^{\circ}$ getrockneter Hemimellithsäure verloren bei 200 bis $220^{\circ} 0,1176 \mathrm{~g} \mathrm{H}_{2} \mathrm{O}$.

$0,9780 \mathrm{~g}$ bei $110^{\circ}$ getrockneter Hemimellithsäure verloren hei 200 bis $220^{\circ} 0,0806 \mathrm{H}_{2} \mathrm{O}$.

\begin{tabular}{ccc}
$\begin{array}{c}\text { Berechnet für } \\
1 \mathrm{H}_{2} \mathrm{O}\end{array}$ & \multicolumn{2}{c}{ Gefunden } \\
8,55 & 8,557 & 8,13
\end{tabular}

Nach dem Erkalten erhält man das Hemimellithsäureanhydrid als krystallinische Masse, welche bei $196^{\circ}$ schmilzt und ohne weitere Reinigung bei der Analyse Zahlen giebt, welche der Formel

enteprechen.

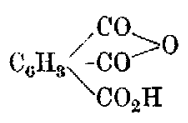

$0,1491 \mathrm{~g}$ gaben $0,3060 \mathrm{CO}_{2}$ und $0,0312 \mathrm{H}_{2} \mathrm{O}$.

$0,1438 \mathrm{~g} . \quad, \quad 0,2944 \mathrm{CO}_{2} \quad, \quad 0,0810 \mathrm{H}_{2} \mathrm{O}$. 
Graebe und Leonhardt, Ueber Hemimellithsäure.

\begin{tabular}{lcrr} 
& Berechnet & \multicolumn{2}{c}{ Gefunden } \\
$\mathrm{C}$ & 56,24 & 55,97 & 55,84 \\
$\mathrm{H}$ & 2,08 & 2,31 & 2,38
\end{tabular}

Das Anhydrid lässt sich bis gegen $300^{\circ}$ erwärmen, ohne sich zu zersetzen. Erst wenn die Temperatur höher wie $300^{\circ}$ steigt, fängt es an sich in Phtalsäureanhydrid und Kohlensäure zu zerlegen. Erhitzt man es in einer Retorte in einem Oelbade auf $310-320^{\circ}$, so sublimiren Nadeln von Phtalsäureanhydrid; aber das Zerfallen bedarf einer längeren Zeit. Destillirt man es rasch aus einer Retorte, so geht ein Gemenge von Phtalsäureanhydrid und unverändertem Hemimellithsäureanhydrid über, während ein harziger Rückstand in der Retorte bleibt. Benzoësäure trat weder beim langsamen Erhitzen anf $300-320^{\circ}$ noch beim raschen Ueberdestilliren auf.

Beim Kochen mit Wasser geht das Anhydrid wieder in Hemimellithsäure über. Salze dieses Anhydrids werden, wie unten angegeben, aus den sauren Salzen der Hemimellithsäure durch Erhitzen erhalten.

Besonders charakteristisch für die Hemimellithsäure ist die Bildung des schwerlöslichen Monokaliumsalzes. Setzt man zu einer kalt gesättigten und nicht übersättigten Lösung derselben in Wasser eine Chlorkaliumlösung hinzu, so scheiden sich sofort Blättchen des Kaliumsalzes aus. Es erfolgt dies sowohl durch concentrirte wie verdünnte Lösungen von Chlorkalium. Diese Eigenschaften unterscheidet die Hemimellithsäure von der Phtalsäure, deren kalt gesättigte Lösungen nicht durch Kaliumchlorid gefällt werden, und erlaubt auch eine recht scharfe Trennung, wenn die Phtalsãuremengen nicht relativ zu gross sind. $0,1808 \mathrm{~g}$ Hemimellithsäure und $0,1742 \mathrm{~g}$ Phtalsäureanhydrid wurden in $5 \mathrm{ccm}$ heissem Wasser gelöst und $5 \mathrm{ccm}$ einer 15 procentigen Chlorkaliumlösung zugesetzt; es krystallisirten $0,2100 \mathrm{~g}$ fast reines hemimellithsaures Kali aus. Vollkommen rein wurde es durch nochmaligẹs Krystallisiren aus Wasser erhalten, wie aus dem Schmelzpunkt der daraus ausgeschiedenen Säure hervorging. Die kalt gesättigte 
Hemimellithsäurelösung wird auch durch die Lösungen von Kaliumsulfat, Ammoniumchlorid, Chlorcalcium und Chlorbaryum, aber nicht durch Natriumchlorid von 10 pC. gefällt. Weniger gut lässt sich Hemimellithsäure von Phtalsäure durch Salzsäure unterscheiden und trennen, da auch aus kalt gesättigter Phtalsäurelösung auf Zusatz von concentrirter Chlorwasserstoffisäure sich beim Stehen Krystalle ausscheiden.

Monokatiumsalz, $\mathrm{C}_{3} \mathrm{H}_{5} \mathrm{O}_{6} \cdot \mathrm{K}+2 \mathrm{H}_{2} \mathrm{O}$. Dieses Salz, welches man bei der Darstellung der Hemimellithsäure gewinnt, kann man auch durch Auflösen der Säure in der berechneten Menge Kaliumcarbonat oder Fällen der Säure mit Chlorkalium oder Kaliumacetat darstellen. Es krystallisirt in Blättchen und ist in kaltem Wasser wenig löslich; bei $17^{0}$ lösen 100 Theile Wasser nur $0,587 \mathrm{~g}$ desselben (auf wasserfreies berechnet); Gegenwart von ChIorkalium oder Kaliumsulfat vermindert die Löslichkeit. In heissem Wasser ist es viel löslicher, in ungefähr zehn Theilen; Aether löst es kaum.

$0,7248 \mathrm{~g}$ lufttrocknes Salz verloren bei $100^{\circ} 0,0900 \mathrm{H}_{2} \mathrm{O}$.

Berechnet fïr Gefunden

$2 \mathrm{H}_{\mathbf{2}} \mathrm{O}$

12,74

$0,3646 \mathrm{~g}$ bei $100^{\circ}$ getrocknetes Salz, gaben $0,1276 \mathrm{~K}_{\mathbf{8}} \mathrm{SO}$.

$0,3222 \mathrm{~g} \quad 100^{\circ} \quad, \quad " \quad$ " $\quad 0,1100 \mathrm{~K}_{\mathbf{2}} \mathrm{SO}_{4}$.

Berechnet für Gefunden

$\mathrm{C}_{8} \mathrm{H}_{5} \mathrm{O}_{6} \mathrm{~K}$

K $15,72 \quad 1 \check{0}, 6915, \check{0}$

Erhitzt man das auf $100^{\circ}$ getrocknete Salz auf $200^{\circ}$, so verliert es ohne zu schmelzen ein weiteres Molekül Wasser.

$0,2584 \mathrm{~g}$ verloren $0,0194 \mathrm{H}_{2} \mathrm{O}$.

$\begin{array}{ccc} & \text { Berechnet } & \text { Gefunden } \\ \mathrm{1H}_{2} \mathrm{O} & 7,26 & 7,60\end{array}$

Es beruht dies offenbar auf Anhydridbildung und spricht dafür, dass in dem Kaliumsalz das Kalium nicht in dem mittleren Carboxyl enthalten ist, da sonst diese Wasserabspaltung nicht eintreten würde: 
224 Graebe und Leonhardt, Ueber Hemimellithsäure.

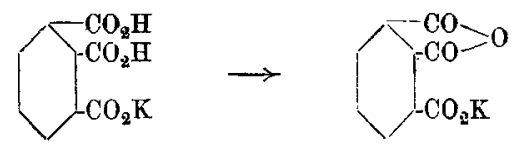

Dass diese Auffassung richtig ist, geht auch daraus hervor, dass das auf $200^{\circ}$ erhitzte Salz sich mit Benzol condensiren lässt.

Um ein Dikaliumsalz darzustellen, wurde Hemimellithsäure mit genau einem Molekül Kaliumcarbonat in Wasser gelöst. Aus der wässrigen Lösung schied sich aber das schwerlösliche Monokaliumsalz aus und in Lösung blieb das neutrale Salz.

Das Trikaliumsalz, $\mathrm{C}_{9} \mathrm{H}_{3} \mathrm{O}_{6} \mathrm{~K}_{3}$, erhält man durch Auflösen der Hemimellithsäure in der berechneten Menge Kaliumcarbonat; es ist sehr leicht löslich und nur aus ganz concentrirter Lösung krystallisirt es über Schwefelsäure in Nadeln.

$0,3376 \mathrm{~g}$, bei $125^{\circ}$ getrocknet, gaben $0,2710 \mathrm{~K}_{2} \mathrm{SO}_{4}$.

$\begin{array}{ccc} & \text { Berechnet } & \text { Gefunden } \\ \mathrm{K} & 36,11 & 35,98\end{array}$

Das neutrale Baryumsalz wurde schon von Baeyer untersucht. Baeyer hat funf Moleküle Krystallwasser in demselben gefunden, von denen bei $160^{\circ}$ nur ein Molekül entweicht. Unsere Analysen stimmen für das im Exsiccator getrocknete Salz besser mit der Formel $\left(\mathrm{C}_{9} \mathrm{H}_{3} \mathrm{O}_{6}\right)_{2} \mathrm{Ba}_{3}+6 \mathrm{H}_{2} \mathrm{O}$, von denen in Uebereinstimmung mit Baeyer nur eins bei $160^{\circ}$ entweicht. 100 Theile Wasser lösen bei $25^{\circ} 0,36$ Theile desselben; in heissem Wasser ist es nur sehr wenig mehr löslich.

Das neutrale Silbersalz ist schon in der vorhergehenden Arbeit beschrieben; es wird am einfachsten erhalten durch Auflösen des Monokaliumsalzes in verdüuntem Ammoniak und Fällen mit Silbernitrat. Versetzt man direct eine Lösung des Monokaliumsalzes mit salpetersaurem Silber, so scheidet sich immer ein Salz ans, welches mehr Silber enthält, als einem Atom entspricht. Kocht man einige Zeit die Lösung des 
Kaliumsalzes mit uberschüssigem Silbernitrat, so bildet sich ein schwer lösliches Salz, welches einem Disilbersalz entspricht.

$0,1378 \mathrm{~g}$ gaben $0,0702 \mathrm{Ag}$.

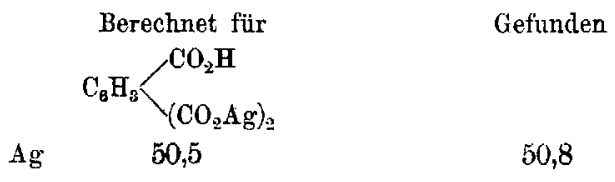

Esterbildung der Hemimellithsäure.

Die interessanten Untersuchungen V. Meyer's über Esterbildung aromatischer Sätren finden eine Bestätigung in dem Verbalten der Hemimellithsäure. Aus derselben erhält man direct nur den Dimethylester

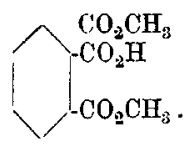

Behandelt man aber an Stelle der Säure das Hemimellithsäureanhydrid mit Methylalkohol und Chlorwasserstoff, so bildet sich ein Gemenge eines Mono- und eines Trimethylesters. Dieser Monomethylester entsteht als alleiniges Product, wenn man das Anbydrid mit Methylalkohol kocht. Unterwirft man ihn dann der Einwirkung von Methylalkohol und Salzsüure, so liefert er nur Trimethylester und nicht das obige Dimethylderivat. Demnach muss man wohl annehmen, dass Methylalkohol das Anhydrid in einen Ester verwandelt, welcher das Methyl in dem mittleren Carboxyl enthält:

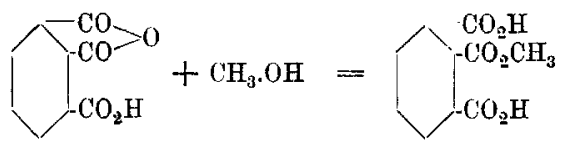

Derselbe liefert dann bei weiterer Esterificirung den Trimethylester. Hiermit stimmt überein, dass der Trimethylester, der auch aus dem Silbersalz erhalten werden kann, beim Verseifen mit zwei Molekulen Aetznatron in glatter Weise in denselben Monomethylester übergeführt wird, entsprechend den Beobachtungen von V. Meyer, nach denen die Ester, die 
sich schwer bilden, sich auch schwer verseifen. Wir haben noch Versuche angestellt, einen isomeren Monomethylester darzustellen, der das Methyl in einem der äusseren Carboxyle enthält und zu diesem Zweck das Monokaliumsalz mit Jodmethyl sowohl auf $100^{\circ}$ wio auf $150^{\circ}$ erhitzt; das Kaliumsalz wurde aber nicht verändert. Das Silbersalz konnte nicht benutzt werden, da wir das Monosilbersalz nicht rein erhielten.

$$
\begin{aligned}
& \text { Monomethylester, } \\
& \mathrm{C}_{6} \mathrm{H}_{5}<-\mathrm{CO}_{2} \mathrm{CH}_{3} . \\
& \mathrm{CO}_{2} \mathrm{H}
\end{aligned}
$$

Behandelt man Hemimellithsäureanhydrid mit 20 Theilen Methylalkohol and Chlorwasserstoffgas bei niederer Temperatur, so scheidet dieser Ester sich sofort aus, lässt man ihn aber mit dieser Lösung stehen, so geht er allmählich in den Trimethylester über. Zweckmässiger stellt man ihu durch Kochen des Anhydrids mit Methylalkohol während $1 / 2-1$ Stunde dar. Man dampft zur Trockne und krystallisirt aus heissem Wasser. In kaltem Wasser und kaltem Methylalkohol ist er schwerer löslich wie Hemimellithsäure. Aus den heissen Lösungen erhält man ihn in Nadeln krystallisirt. Er schmilzt bei $203-205^{\circ}$, und es tritt eine complicirtere Zersetzung ein. Der Verlust beträgt mehr wie ein Molekül Wasser, und die Analysen zeigten, dass ein Gemenge vorlag.

Die Analyse des Monomethylesters entspricht obiger Formel. $0,1466 \mathrm{~g}$ gaben $0,2880 \mathrm{CO}_{2}$ und $0,0490 \mathrm{H}_{2} \mathrm{O}$.

$\begin{array}{ccc} & \text { Berechnet } & \text { Gefunden } \\ \mathrm{C} & 53,50 & 533,64 \\ \mathbf{H} & 3,50 & 3,67\end{array}$

Dimethylester, $\mathrm{C}_{6} \mathrm{H}_{3}-\frac{C}{\mathrm{CO}_{2} \mathrm{CH}} \mathrm{CO}_{3}$
$\mathrm{CO}_{2} \mathrm{CH}_{3}$.

Ein Theil bei $100^{\circ}$ getrocknete Hemimellithsäure wurde in 20 Theilen Methylalkohol gelöst und mit Salzsäure gesättigt. 
Nachdem die Lösung über Nacht gestanden hatte, wurde sie concentrirt, es schieden sich Krystalle aus, welche bei $145^{\circ}$ schmelzen, sich leicht in Alkohol, ziemlich gut in heissem Wasser und wenig in kaltem Wasser lösen und sauer reagiren. Natronlauge löst sie leicht. in der Kälte. Der Ester krystallisirt in Nadeln.

$0,1730 \mathrm{~g}$ gaben $0,3504 \mathrm{CO}_{2}$ und $0,0648 \mathrm{H}_{2} \mathrm{O}$.

$0,1590 \mathrm{~g} \quad, \quad 0,3158 \mathrm{CO}_{2} \quad, \quad 0,0603 \mathrm{H}_{2} \mathrm{O}$.

\begin{tabular}{ccrr} 
& Berechnet & \multicolumn{3}{c}{ Gefunden } \\
C & $\check{\jmath} \check{0}, 46$ & $\tilde{0} \check{0}, 24$ & 55,19 \\
H & 4,20 & 4,16 & 4,22
\end{tabular}

Dieser Ester ist sehr beständig und zersetzt sich selbst nicht beim Destilliren. Um nachzuweisen, dass entsprechend der Theorie von V. Meyer bei der Esterbildung das mittlere Carboxyl nicht in Reaction tritt, haben wir durch Lösen in Ammoniak und Fällen mit Silbernitrat das Silbersalz dargestellt und dieses vorsichtig der Destillation unterworfen. Ein Theil verpufft, doch konnte neben Benzol und Diphenyl eine geringe Menge eines niedrig schmelzenden Körpers erhalten werden, der beim Verseifen eine über $300^{\circ}$ schmelzende Säure lieferte, welche in Wasser schwer löslich war und in Nadeln krystallisirte, also die Eigenschaften der Isophtalsäure besitzt.

Trimethylester, $\mathrm{C}_{6} \mathrm{H}_{3}\left(\mathrm{CO}_{2} \mathrm{CH}_{3}\right)_{3}$. Derselbe lässt sich aus dem Silbersalz und Jodmethyl darstellen; es wurde auf 120 bis $125^{\circ}$ während einiger Stunden erhitzt. Ferner wurde er erhalten, als ein Theil Hemimellithsäureanhydrid und 20 Theile Methylalkohol mit Chlorwasserstoff gesättigt wurden und dann mebrere Tage stehen blieben. Dabei ging der grösste Theil des anfangs ausgeschiedenen Monomethylesters in Lösung. Beim langsamen Verdampfen der filtrirten Lösung schieden sich Krystalle aus, welche in Zusammensetzung und Eigenschaften mit dem aus dem Silbersalz erhaltenen Aether übereinstimmen. Er kann auch aus dem Monomethylester durch Behandeln mit Methylalkohol und Chlorwasserstoff erhalten werden.

Der Trimethylester schmilzt bei $100^{\circ}$, ist in Wasser und 
Alkalien in der Kälte nicht löslich, löst sich leicht in Alkohol und Aether.

$0,1090 \mathrm{~g}$ gaben $0,2272 \mathrm{CO}_{2}$ und $0,0480 \mathrm{H}_{2} \mathrm{O}$.

$0,1456 \mathrm{~g} \quad, \quad 0,3052 \mathrm{CO}_{2} \quad, \quad 0,0620 \mathrm{H}_{2} \mathrm{O}$.

\begin{tabular}{ccrr} 
& Berechnet & \multicolumn{2}{c}{ Gefunden } \\
C & 57,14 & 56,85 & 57,15 \\
H & 4,76 & 4,99 & 4,74
\end{tabular}

Beim Kochen des Trimethylesters mit zwei Molekülen Aetznatron in wässriger Lösung und nachherigem Fällen mit Salzsäure wird der Monomethylester, aus dem er sich bildet, zurückerhalten.

Imid der Hemimellithsäure,

$$
\mathrm{C}_{8} \mathrm{H}_{9} \div \underset{\mathrm{CO}_{2} \mathrm{H}}{\mathrm{CO}}>\mathrm{NH} \text {. }
$$

Dasselbe lässt sich genau so darstellen wie Phtalimid. Leitet man in Hemimellithsäureanhydrid, welches auf 200 bis $220^{\circ}$ erhitzt ist, Ammoniak ein, so wird auf einmal die Masse fest. Krystallisirt man das Product aus Wasser, so erhält man das Hemimellithimid in Form farbloser Nadeln, welche bei $247^{\circ}$ (corr.) schmelzen.

$0,1446 \mathrm{~g}$ gaben $0,2996 \mathrm{CO}_{2}$ und $0,0368 \mathrm{H}_{2} \mathrm{O}$.

$0,1490 \mathrm{~g} \quad, 11 \mathrm{~cm}$ Stickgas bei $30^{\circ}$ und 731,5 mm Druck.

$\begin{array}{ccc} & \text { Berechnet } & \text { Gefunden } \\ \mathrm{C} & 56,54 & 56,50 \\ \mathrm{H} & 2,62 & 2,82 \\ \mathrm{~N} & 7,33 & 7,52\end{array}$

In kaltem Wasser ist es sehr wenig, leichter in heissem Wasser löslich; Alkohol und Eisessig lösen es nicht reichlich. Alkalien und Alkalicarbonate lösen es schon in der Kälte und beim Kochen dieser Lösungen wird Hemimellithsäure regenerirt. Durch die leiehte Löslichkeit in Alkalicarbonaten unterseheidet es sich rom Phtalimid. Das Hemimellithimid hat den Charakter einer zweibasisehen Säure, da anch der Imidwasserstoff leicht durch Metalle ersetzt wird. 
Graebe und Leonhardt, Ueber Hemimellithsäure. 229

Das Silbersalz,

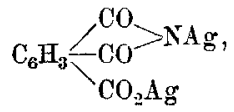

entsteht durch Fällen des in Ammoniak gelösten Imids mittelst Silbernitrat als weisser Niederschlag, welcher bei $100^{\circ}$ obiger Formel entspricht.

$0,1605 \mathrm{~g}$ gaben $0,0860 \mathrm{Ag}$.

$$
\begin{array}{ccc} 
& \text { Berechnet } & \text { Gefunden } \\
\mathrm{Ag} & 53,33 & 53,57
\end{array}
$$

Das Calciumsalz, $\mathrm{C}_{9} \mathrm{H}_{3} \mathrm{O}_{4} \mathrm{Ca}+1 \frac{1}{2} \mathrm{H}_{2} \mathrm{O}$, wurde durch Erwärmen einer wässrigen Lösung des Imids mit Marmorpulver und Concentriren der Lösung dargestellt. Beim Erkalten scheiden sich farblose Blättchen aus.

$0,2380 \mathrm{~g}$ verloren bei $150^{\circ} 0,0250 \mathrm{H}_{2} \mathrm{O}$ und gaben $0,0516 \mathrm{CaO}$.

$\begin{array}{lcc} & \text { Bèrechnet } & \text { Gefunden } \\ 1{ }^{1} / \mathrm{H}_{2} \mathrm{O} & 10,55 & 10,50 \\ \mathrm{Ca} & \mathbf{1 7 , 4 7} & \mathbf{1 7 , 3 0}\end{array}$

Condensation von Hemimellithsüure mit Benzol.

Entsprechend der Synthese von o-Benzoylbenzoësäure aus Phtalsäure lzönnen aus Hemimellithsäureanhydrid und Benzol durch Einwirkung von Chloraluminium zwei Säuren entstehen:

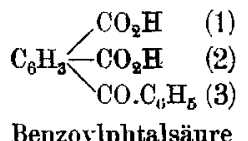

Benzoylphtalsäure

$$
\mathrm{C}_{6} \mathrm{H}_{3}=\underset{\mathrm{CO}_{2} \mathrm{H}}{\mathrm{CO}_{2} \mathrm{H}}
$$

Benzoylisophtalsäure.

Die erste bildet sich als Hauptproduct bei Anwendung von Hemimellithsäureanhydrid; doch wird immer ein Theil derselben durch Condensation mit einem zweiten Molekül Benzol in die beiden Dibenzoylbenzoësäuren $1,2,3$ und $1,2,6$
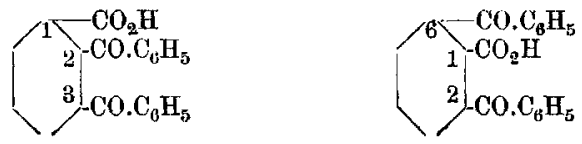

verwandelt, indem offenbar vorher die Benzoylphtalsäure in ihr Anhydrid ubergeht. Welche der letzteren entsteht, hängt 
wesentlich vom Verlaufe der Reaction ab. Die Benzoylisophtalsăure wurde bei diesen Versuchen nicht erhalten.

Wendet man aber an Stelle des Anhydrids der Hemimellithsäure das Anhydrid des Monokaliumsalzes,

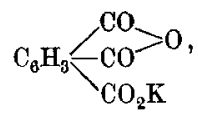

an, so entsteht neben Benzoylphtalsäare und der 1,2,3-Dibenzoylbenzoësänre auch die Benzoylisophtalsäure und bildet etwa die Hälfte des Products.

$$
\begin{gathered}
\text { Benzoylphtalsäure, } \\
\mathrm{C}_{8} \mathrm{H}_{3}<-\mathrm{CO}_{8} \mathrm{H} \\
\mathrm{CO}_{8} \mathrm{C}_{8} \mathrm{H}_{5} .
\end{gathered}
$$

Diese Säure bildet sich am reichlichsten, wenn die Reactionsdauer eine kurze ist. Ein Theil feingepulvertes Hemimellithsäureanhydrid wurden mit $1-1 \frac{1}{2}$ Theilen gepulvertem Chloraluminium und vier bis fünf Theilen Benzol kalt gut durchgeschüttelt und dann auf dem Wasserbade am Rückflusskühler erwärmt. Man unterbricht, sowie die stürmische Reaction nachlässt, was gewöhnlich nach 20-25 Minuten der Fall war. Nach dem Abkühlen giebt man vorsichtig durch den Kühler Wasser hinzu und trennt das Benzol von den wässrigen Lösungen und der ausgeschiedenen festen Substanz. Im Benzol ist so gut wie nichts gelöst. Das erhaltene Product wurde mit verdünnter Salzsäure und viel Wasser erwärmt. Man lässt auf $40-50^{\circ}$ erkalten und filtrirt von einer harzigen Masse, welche wesentlich aus der 1,2,6-Dibenzoylbenzoës̈äure besteht. Die wässrige Lösung wird etwas concentrirt und erkalten gelassen. Es scheidet sich die Benzoylphtalsäure in Form farbloser Nadeln aus. Zum Reinigen wurden sie nochmals aus heissem Wasser krystallisirt.

Die Benzoylphtalsäure krystallisirt mit ẹinem Molekül Krystallwasser, welches bei $100^{\circ}$ weggeht; bei dieser Temperatur beginnt aber schon Anhydridbildung, der Wasserverlust 
Graebe und Leo nhardt, Ueber Hemimellithsäure. 231

war daher etwas zu gross und bei der Verbrennung wurde dementsprechend zu viel Kohlenstoff gefunden.

$3,9834 \mathrm{~g}$ verioren bei $100^{\circ} 0,356 \mathrm{H}_{2} \mathrm{O}$.

Berechnet für

$1 \mathrm{H}_{\mathbf{8}} \mathrm{O}$

6,25
Gefunden

8,94

$0,1310 \mathrm{~g}$ gaben $0,3228 \mathrm{CO}_{2}$ and $0,0444 \mathrm{H}_{\mathrm{q}} \mathrm{O}$.

$0,1480 \mathrm{~g} \quad, \quad 0,3690 \mathrm{CO}_{2} \quad, \quad 0,0494 \mathrm{H}_{2} \mathrm{O}$.

Berechnet für

Gefunden

$$
\mathrm{C}_{15} \mathrm{H}_{10} \mathrm{O}_{5}
$$

$\begin{array}{llll}\mathrm{C} & 66,67 & 67,20 & 67,32\end{array}$

H $\quad 3,70 \quad 3,80 \quad 3,76$

Das durch Erhitzen auf 145-150 erhaltene Anhydrid gab aber bei der Analyse richtige Zahlen.

$0,1580 \mathrm{~g}$ gaben $0,4130 \mathrm{CO}_{2}$ und $0,0504 \mathrm{H}_{2} \mathrm{O}$.

Berechuet für

$\mathrm{C}_{15} \mathrm{H}_{8} \mathrm{O}_{4}$

$\mathrm{C}$

H
71,43

3,17
Gefinden

71,28

3,54

Die Benzoylphtalsäure selbst zeigt keinen bestimmten Schmelzpunkt; schon etwas über $100^{\circ}$ beginnt sie weich $\mathrm{zu}$ werden, bei $130^{\circ}-140^{\circ}$ beobachtet man Aufschäumen und die Substanz wird dann bei $183^{\circ}$ (corr.), d. h. bei dem Schmelzpunkt des Anhydrids, vollständig flüssig. Sie ist in heissem Wasser leicht und in kaltem wenig löslich. Alkohol löst sie reichlich, Aether weniger gut und Benzol sehr wenig. In concentrirter Schwefelsăure löst sie sich in der Kälte mit hellgelber Farbe, welche beim Erwärmen etwas dunkler wird, und bei ungefähr $115^{\circ}$ nimmt die Lösung eine grünliche Färbung an und wird bei kurzem Erhitzen auf $145-150^{\circ}$ schön olivengrün. Die Benzoylphtalsäure ist alsdann in 1-Anthrachinoncarbonsäure verwandelt :
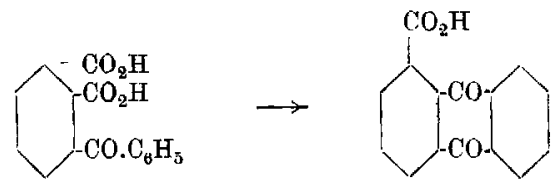
Nachdem der Farbenumschlag eingetreten ist, giesst man in Wasser. Die ausgeschiedene Säure wurde aus heissem Wasser umkrystallisirt, in dem sie aber schwer löslich ist. Es wurden hellgelbe Nadeln erhalten, welche bei $293-294^{\circ}$ (corr.) schmelzen. Mit Natronlauge und Zinkstaub entsteht eine blutrothe Lösung, welche sich an der Luft wieder entfärbt. Das Baryumsalz dieser Anthrachinoncarbonsäure ist in kaltem Wasser sehr schwer löslich. Aus heissem Wasser, in dem es etwas besser löslich ist, erhält man es in Nadeln. Diesen Eigenschaften nach ist sie mit der von Liebermann und Bischoff ${ }^{5}$ ) aus $\gamma$-Anthracencarbonsäure durch 0xydation erhaltenen identisch. Der Schmelzpunkt wurde uncorrigirt von denselben zu $285^{\circ}$ angegeben. Die Bildung dieser Säure aus Hemimellithsäure beweist nun bestimmt, dass das Carboxyl die Stelle 1 einnehmen muss und ist es deshalb richtig, die bisher gebrauchte $\mathrm{Be}$ zeichnung $\gamma$-Anthrachinoncarbonsäure durch 1-Anthrachinoncarbonsäure zu ersetzen.

Zur weiteren Charakterisirung derselben haben wir den Aethylester dargestellt. Die 1-Anthrachinoncarbonsäure wurde mit etwas mehr als einem Molekül Phosphorchlorid gemischt und erwärmt und zwar schliesslich bis gegen 200. Das erhaltene feste Product wurde direct mit Alkohol gekocht, dann die Lösung nach dem Erkalten in Natriumcarbonat gegossen und das Ungelöste aus Alkohol krystallisirt. Man erhält gelbe Nadeln, welche leicht in heissem, weniger in kaltem Alkohol löslich sind und bei $169^{\circ}$ schmelzen. Der Schmelzpunkt des Aethylesters der 2-Anthrachinoncarbonsäure liegt bei $147^{\circ}$.

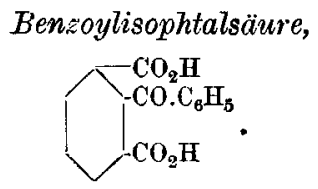

Zur Darstellung derselben wird genau so verfahren wie bei der Benzoylphtalsäure angegeben, nur an Stelle des Anhydrids

5) Ber. d. deutsch. chem. Ges. 13, 49. 
der Hemimellithsäure das Anhydrid des Monokaliumsalzes angewandt. Aus der wässrigen Lösung scheidet sich zuerst eine viel schwerer lösliche Säure aus. Die Analysen der bei $100^{\circ}$ (I.) und der bei $200-210^{\circ}$ (II.) getrockneten Säure gaben übereinstimmend Zahlen, die obiger Formel entsprechen.

I. $0,1316 \mathrm{~g}$ gaben $0,3216 \mathrm{CO}_{2}$ und $0,0484 \mathrm{H}_{2} \mathrm{O}$.

II. $0,1194 \mathrm{~g} \quad " \quad 0,2916 \quad \mathrm{CO}_{2} \quad, \quad 0,0430 \mathrm{H}_{2} \mathrm{O}$.

\begin{tabular}{ccrr} 
& Berechnet & \multicolumn{2}{c}{ Gefunden } \\
\cline { 3 - 4 } C & 66,67 & I. & II. \\
H & 3,70 & 66,59 & 66,60 \\
& & 4,08 & 4,00
\end{tabular}

Die Benzoylisophtalsäure ist sehr wenig in kaltem Wasser, etwas reichlicher in heissem Wasser, leicht in Alkohol und nicht sehr reichlich in Aether löslich. Sie lüsst sich gut aus verdiunntem Alkohol krystallisiren und wird in Nadeln oder Säulen erhalten. Sie schmilzt bei $260^{\circ}$.

Mit concentrirter Schwefelsäure einige Minuten auf 140-150 erwärmt, verwandelt sie sich wie die Benzoylphtalsäure in 1-Anthrachinoncarbonsäure. Man beobachtet dabei denselben Farbenübergang von gelb in grün. In Betrefi der Eigenschaften der so erhaltenen Anthrachinoncarbonsäure verweisen wir auf das oben Angeführte. Diese Condensation entspricht genau obiger Constitutionsformel.

$$
\text { Dibenzoylbenzoësäure, 1-2-3-, }
$$

Diese Säure entsteht als Hauptproduct bei der Einwirkung von Benzol und Aluminiumchlorid auf Hemimellithsäureanhydrid, wenn man, nachdem die erste stïrmische Reaction vorbei ist, noch fünt bis sechs Stunden am aufsteigenden Kühler erwärmt. Hat man dann das Reactionsproduct mit heissem Wasser behandelt und auf $40-50^{\circ}$ abkühlen lassen, so bleibt ein zum grössten Theil krystallinischer Rückstand, welcher, ans Fisessig 
234 Graebe und Leonhardt, Ueber Hemimellithsäure.

krystallistirt, eine bei $208^{\circ}$ schmelzende Säure liefert, deren Analyse einer Dibenzoylbenzoësäure entspricht.

$0,1388 \mathrm{~g}$ gaben $0,3892 \mathrm{CO}_{2}$ und $0,0532 \mathrm{H}_{2} \mathrm{O}$.

Berechnet

C

$\mathrm{H}$
76,36

4,24
Gefunden

76,47

4,33

Diese Säure ist in Wasser so gut wie unlöslich, in Alkohol und Aether löst sie sich leicht.

Um zu entscheiden, ob die beiden Benzoylgruppen unter sich die Stellungen 1,2 oder 1,3 haben, wurde dieselbe der trocknen Destillation unterworfen. Es ging neben etwas unveränderter Säure ein in wässriger Natronlauge unlösliches Product uber. Dasselbe aus Alkohol krystallisirt lieferte bei $115^{\circ}$ schmelzende Blättchen. Dass es bestimmt von dem 1,3-Dibenzoylbenzol, welches bei $99,5^{0}$ schmilzt, verschieden ist, geht aucb daraus hervor, dass es beim Erwärmen mit Hydroxylamin in alkoholischer Iösung kein Oxim lieferte. Auch dem 1,2-Dibenzoylbenzophenon, welches nach $Z$ incke bei $145^{\circ}$ schmilzt, entspricht es also nicht. Es zeigte sich dann mit Phtalophenon identisch; Schmelzpunkt, Krystallform und Löslichkeit stimmen. Erwărmt man es mit alkoholisehem Kalihydrat, so geht es in Lösung und Säuren fällen es dann wieder aus. Das Phtalophenon kann sich nun sehr gut aus einer Säure bilden, welche die beiden Benzoylgruppen in benachbarter Stellung enthält; es würde das o-Dibenzoylbenzol, welches durch Abspalten von Kohlensäure entsteht, sich zu Phtalophenon umlagern. Keinenfalls würde es sich aber aus der isomeren Säure, welche die beiden Benzoyle in Metastellung enthalt, bilden, da das Isophtalophenon unzersetzt destillirt.

Möglicherweise ist die Dibenzoylbenzoësäure selbst schon durch Umlagerung in Phtalophenoncarbonsäure,
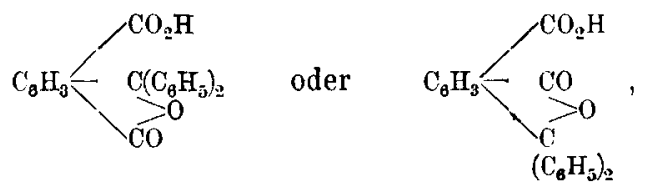
übergegangen. Versuche, um diese Frage zu entscheiden, haben wir nicht unternommen, wir nehmen daher die der Bildung einfacher entspréchende Formel an, nach der sie eine Dibenzoylbenzoësäure ist.

Dibenzoylbenzoёsäure, 1-2-6,

$$
\int_{\mathrm{CO} . \mathrm{C}_{6} \mathrm{H}_{5}}^{-\mathrm{CO}_{6} \mathrm{C}_{6} \mathrm{H}_{5}} \text {. }
$$

Dieses Dibenzoylderivat entsteht neben dem vorher beschriebenen bei Anwendung von Hemimellithsäureanhydrid und zwar wesentlich bei kurzer Reactionsdauer. Das in Wasser unlösliche Product wurde in Natronlauge gelöst und dann nach vollständigem Erkalten mit Salzsäure gefällt, wobei sich die Säure, wenn man ganz kalt fällt, krystallinisch, sonst leicht harzig ausscheidet. In Alkohol und Aether ist sie leichter wie die isomere Säure löslich, aus diesen Lösungen scheidet sie sich aber ölig aus. Ihr Schmelzpunkt liegt ungefähr bei $100^{\circ}$.

$0,1238 \mathrm{~g}$ gaben $0,3460 \mathrm{CO}_{2}$ und $0,0530 \mathrm{H}_{2} \mathrm{O}$.

$\begin{array}{ccc} & \text { Berechnet } & \text { Gefunden } \\ \text { C } & 76,66 & 76,22 \\ \text { H } & 4,24 & 4,76\end{array}$

\section{Fluoresceïne der Hemimellithsäure.}

Das Anhydrid der Hemimellithsäure kann bei der Condensation mit Resorcin zwei Derivate bilden, je nachdem die Condensation mit dem Carbonyl 1 oder 2 erfolgt. Kommt nun dem Fluoresceïn, wie dies nach den in der letzten Zeit publicirten Arbeiten in der That wahrscheinlicher ist, die Chinonformel za, so kann das eine jener Fluoresceïne durch Wasserverlust sich in ein Anhydrid verwandeln. Wir haben nun gefunden, dass beim Erhitzen von Hemimellithsäureanhydrid mit Resorcin in der That zwei Fluoresceincarbunsäuren entstehen, von denen die eine in ein Anhydrid übergeht:

Annalen der Chemie 290. Bd. 


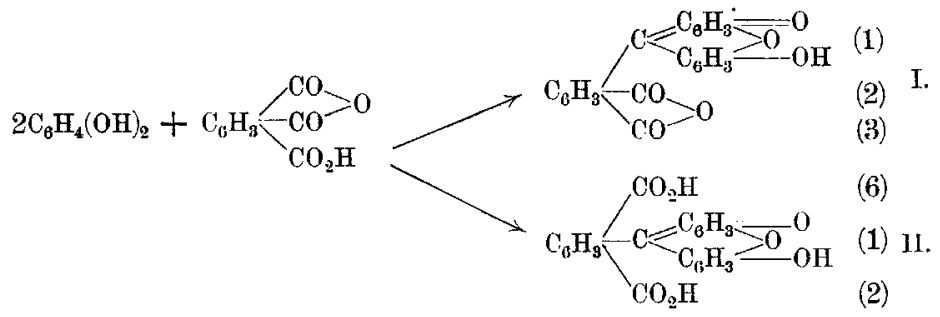

Die Beobachtung, dass ein Anhydrid auftritt, welches genau wie Fluoresceïn gefärbt ist, lässt sich nun zweifellos besser durch die Chinonformel erklären.

Ein Theil Hemimellithsäureanhydrid wurde mit 1,3 Theilen Resorcin während drei Stunden auf $200^{\circ}$ erhitzt. Die erhaltene Masse wurde pnlverisirt und dann wiederholt mit Wasser ausgekocht. Ein Theil des entstandenen Productes geht in Lösung und scheidet sich beim Erkalten in feinen gelben Nadeln aus.

Der unlösliche Theil wurde in Natriumcarbonat gelöst, aus dem Filtrat mit Salzsäure gefällt und in Form einer dem Fluoresceïn sehr ähnlichen röthlichgelben, krystallinischen Substanz erhalten. Bei $150^{\circ}$ getrocknet und analysirt, ergiebt sie die der Formel I entsprechende Zusammensetzung.

$0,1576 \mathrm{~g}$ gaben $0,4066 \mathrm{CO}_{\mathfrak{\varepsilon}}$ und $0,0450 \mathrm{H}_{2} \mathrm{O}$.

$0,1612 \mathrm{~g} \quad, \quad 0,4134 \mathrm{CO}_{2} \quad, \quad 0,0458 \mathrm{H}_{2} \mathrm{O}$.

\begin{tabular}{lcrr} 
& Berechnet für & \multicolumn{2}{c}{ Gefunden } \\
& $\mathrm{C}_{21} \mathrm{H}_{10} \mathrm{O}_{6}$ & & \\
$\mathrm{C}$ & 70,39 & 70,36 & 69,94 \\
$\mathrm{H}$ & 2,79 & 3,23 & 3,11
\end{tabular}

Man kann sie, entsprechend obiger Formel, als das $A n$ hydrid der 3-Fluoresceïncarbonsäure bezeichnen, indem man nur die Stellung des Carboxyls angiebt, welches auch im Hemimellithsäureanhydrid vorhanden ist, während $1 \mathrm{dem}$ mit den drei Benzolen verbundenen Kohlenstoff entspricht. Sie ist in Wasser unlöslich, in Alkohol löst sie sich wenig und mit gelber Farbe. Die Lösungen in Alkalien sind gelbroth und besitzen eine wenig intensive Fluorescenz.

Entsprechend obiger aufgelöster Formel liefert dieses 
Anhydrid beim Erhitzen mit Essigsäureanhydrid bis zum Siedepunkte des letzteren ein Monoacetylderivat. Auf Alkoholzusatz scheiden sich gelbe Krystalle aus, die durch Waschen mit Alkohol von etwas unverändertem Anhydrid befreit werden. Sie schmelzen erst über $300^{\circ}$ und unterscheiden sich von dem nicht acetylirten Körper dadurch, dass sie in Ammoniak und Alkalien in der Kälte unlöslich sind. Erwärmt man sie mit Alkalien, so tritt Verseifung ein.

$0,1532 \mathrm{~g}$ gaben $0,3870 \mathrm{CO}_{2}$ und $0,0464 \mathrm{H}_{2} \mathrm{O}$.

\begin{tabular}{ccr}
\multicolumn{2}{c}{ Berechnet für } & Gefunden \\
$\mathrm{C}_{21} \mathrm{H}_{9} \mathrm{O}_{5}\left(\mathrm{COCH}_{3}\right)$ & $\mathrm{C}_{91} \mathrm{H}_{8} \mathrm{O}_{6}\left(\mathrm{COCH}_{3}\right)_{2}$ & \\
69,00 & 67,88 & 68,90 \\
3,00 & 3,17 & $3,3 \check{0}$
\end{tabular}

Der in heissem Wasser lösliche Theil geht beim Erhitzen auf $150-180^{\circ}$ nicht in ein Anhydrid aber, wir nehmen daher an, dass er der Formel II entsprechend zusammengesetzt sei und bezeichnen ihn als 6 -Fluoresceïncarbonsäure. Er wurde wie das unlösliche Product in Natriumcarbonat gelöst, mit Salzsäure gefällt und zur Analyse längere Zeit auf $150^{\circ}$ erhitzt. $0,1394 \mathrm{~g}$ gaben $0,3424 \mathrm{CO}_{\mathrm{q}}$ and $0,0389 \mathrm{H}_{2} \mathrm{O}$.

$\begin{array}{ccc} & \text { Berechnet für } & \text { Gefunden } \\ & \mathrm{C}_{21} \mathrm{H}_{12} \mathrm{O}_{7} & \\ \mathrm{C} & 67,02 & 66,98 \\ \mathrm{H} & 3,19 & 3,10\end{array}$

Diese Fluoresceincarbonsäure besteht aus röthlichgelben Nadein, schmilzt noch nicht bei $280^{\circ}$, löst sich kaum in kaltem Wasser, besser aber nicht sehr reichlich in heissem Wasser und leicht in Alkohol, welcher eine dunkelbraune Lösung liefert. In Alkalien löst sie sich mit derselben Farbe und Fluorescenz wie das Fluoresceïn, doch ist die Fluorescenz weniger intensiv.

Wendet man an Stelle des Hemimellithsäureanhydrids das Kaliumsalz dieses Anhydrids zur Darstellung der Fluoresceïne an, so erhält man dieselben Körper und zwar kaliumfrei, indem wohl ein Theil der Hemimellithsäure nicht in Reaction tritt und in das Trikaliumsalz übergeht. 
Erhitzt man das Hemimellithsäureanhydrid mit m-Dimethylaminophenol, so wird ein Rhodamin erhalten, welches sich von den anderen Rhodaminen dadurch unterscheidet, dass es mit violettrother Farbe in Alkalien löslich ist.

Ueber Einwirkung von Brom, Chlor und Schwefel auf Fluoren;

von C. Graebe und B. van Mantz.

In der Absicht, eine Reihe von Fluorenderivaten darzustellen, versuchten wir durch Einwirkung von Brom und Chlor bei höherer Temperatur diese Halogene in das Methylen dieses Kohlenwasserstoffs einzuführen. Aus unseren Versuchen ergab sich nun, dass bei der Schmelztemperatur des Fluorens, das heisst bei $113^{\circ}$ sowie bei $150^{\circ}$, das Brom in die Phenylene und nicht ins Methylen eintritt. Wendet man eine höhere Temperatur an, so werden die Wasserstoffe des Methylens eliminirt; aber an Stelle von Bromsubstitutionsproducten entstehen hochmolekulare Kohlenwasserstoffe, unter denen sich das rothe Dibiphenylenäthen,

befindet.<smiles>CCCCC(CCC)=C1CCCC1</smiles>

Einwirkung von Brom bei $115^{\circ}$ und $150^{\circ}$.

Fluoren wurde in einem Oelbade gerade bis zum Schmelzen erhitzt und die Temperatur auf $113-115^{\circ}$ gehalten. Mit Hülfe eines Kohlensäurestromes wurde die zwei Atomen Brom entsprechende Menge gasförmig eingeleitet. Das Product lieferte durch Krystallisation aus Alkohol einen farblosen, bei $101^{\circ}$ schmelzenden Körper, welcher in Alkohol leicht und in Aether und Chloroform sehr löslich ist. Zusammensetzung wie Eigenschaften beweisen, dass er mit dem Bromfluoren identisch ist, welches Hodgkinson und Matthews durch Einwirkung von 